\title{
Perfil de Usuários de Crack no Município de Santos
}

\author{
Luciana Oliveira Villarinho Rodrigues \\ Programa de Pós-Graduação Interdisciplinar em Ciências da Saúde, Universidade \\ Federal de São Paulo, São Paulo, SP, Brasil \\ Grupo de Vigilância Epidemiológica do Estado de São Paulo, Secretaria Estadual de Saúde, \\ São Paulo, SP, Brasil \\ Carlos Roberto de Castro e Silva \\ Departamento de Políticas Públicas e Saúde Coletiva e Programa de Pós-Graduação \\ Interdisciplinar em Ciências da Saúde, Universidade Federal de São Paulo, \\ São Paulo, SP, Brasil \\ Nara Rejane Cruz de Oliveira \\ Departamento de Ciências do Movimento Humano e Programas de Pós-Graduação Ensino \\ em Ciências da Saúde e Interdisciplinar em Ciências da Saúde, Universidade Federal \\ de São Paulo, São Paulo, SP, Brasil \\ Adriana Marcassa Tucci ${ }^{1}$ \\ Departamento de Saúde, Educação e Sociedade e Programa de Pós-Graduação \\ Interdisciplinar em Ciências da Saúde, Universidade Federal de São Paulo,
}

São Paulo, SP, Brasil

\begin{abstract}
Resumo
O uso do crack é uma questão complexa, que exige inovações e articulações intersetoriais na proposição de políticas públicas eficazes. Objetivo: descrever o perfil dos usuários de crack do município de Santos/ SP. Método: estudo transversal e de abordagem quantitativa. Participaram 292 sujeitos, de ambos os sexos, com idade acima de dezoito anos. A amostra foi selecionada por meio da técnica da bola de neve. Utilizou-se para a coleta de dados uma versão adaptada do Addiction Severity Index. Resultados: O perfil dos investigados indica maior prevalência de sujeitos do sexo masculino, solteiros, moradores de rua, com idade média de 35 anos, escolaridade média de 8 anos, problemas relacionados aos vínculos familiar e social, presença marcante de sintomas psicológicos e psiquiátricos, início de consumo de crack na vida adulta, poliusuários de substâncias psicoativas e sem histórico de tratamento. Conclusões: A constatação de tal perfil sugere que as intervenções fundamentadas nas Estratégias de Redução de Danos feitas in loco, com objetivo educativo e preventivo, podem ser as formas mais adequadas de se conseguir inserção e reduzir os danos nesta população. Assim, tais achados podem auxiliar e fortalecer políticas públicas, tal como o consultório na rua, especialmente na área de álcool ou outras drogas.
\end{abstract}

Palavras-chave: Cocaína-crack, transtornos relacionados ao uso de cocaína, características da população.

Endereço para correspondência: Departamento de Saúde, Educação e Sociedade. Rua Silva Jardim, n 136, Térreo, Vl. Mathias, Santos, SP, Brasil 11015-020. Fone/fax: (13) 3878-3731. E-mail: atucci@unifesp.br Este estudo teve o apoio financeiro da Fundação de Amparo à Pesquisa do Estado de São Paulo (FAPESP), processo $n^{0} 2011 / 00188-4$. 


\title{
Profile of Crack Users in Santos City
}

\begin{abstract}
The use of crack is a complex issue, requiring intersectoral innovations and joints in proposing effective public policies. Objective: To describe the profile of crack users in the city of Santos/SP. Method: Cross-sectional study with a quantitative approach. Subjects were 292 crack users, from both sexes, over the age of eighteen. The sample was selected through the snowball technique. It was used for data collection an adapted version of the Addiction Severity Index. Results: The profile of subjects investigated indicates a higher prevalence of male, single, homeless, average age 35, average schooling of 8 years, problems related to family and social ties, high presence of psychological and psychiatric symptoms, beginning of crack consumption in adulthood, poliusers of psychoactive substances and no history of treatment. Conclusions: The finding of such a profile suggests that interventions based on harm reduction strategies made in locus with educational and preventive objective, may be the most appropriate ways to achieve integration and reduce damage in this population. Thus, these findings can help and strengthen public policies, such as Street Outreach Offices, especially in the area of alcohol or other drugs.
\end{abstract}

Keywords: Crack-cocaine, cocaine-related disorders, population characteristics.

\section{Perfil de los Consumidores de Crack en la Ciudad de Santos}

\section{Resumen}

El consumo de crack es un tema complejo, que requiere innovaciones intersectoriales y articulaciones para proponer políticas públicas eficaces. Objetivo: Describir el perfil de los consumidores de crack en la ciudad de Santos/SP. Método: Estudio transversal con enfoque cuantitativo. Participaron 292 sujetos de ambos sexos, mayores de dieciocho años. La muestra fue seleccionada por la técnica de bola de nieve. Fue utilizado para la recolección de datos una versión adaptada del Índice de Severidad de la Adicción. Resultados: El perfil investigado indica una mayor prevalencia de varones, solteros, edad media de 35 años, de escolaridad media de 8 años, las problemas relacionados con los vínculos familiares y sociales, marcada presencia de síntomas psicológicos y psiquiátricos, principio del consumo de crack en la edad adulta, poliusers de sustancias psicoactivas y sin antecedentes de tratamiento. Conclusiones: El hallazgo de un perfil de este tipo sugiere que las intervenciones basadas en las estrategias de reducción de daños hechos en locuo, con objetivo educativo y preventivo, pueden ser los medios más apropiados para lograr la integración y reducir los daños en esta población. Por lo tanto, estos resultados pueden ayudar y fortalecer las políticas públicas, como las equipos de salud callejera, sobre todo en el área de alcohol u otras drogas.

Palabras clave: Cocaína-crack, trastornos relacionados con cocaína, características de la población.

Na atualidade, o uso do crack é uma questão complexa, que envolve a saúde, a segurança e a assistência social, dentre outras instâncias. Dados do II Levantamento Nacional de álcool e Drogas (Laranjeira et al., 2012) demonstram que 2 milhões de brasileiros já usaram cocaína fumada (crack/merla/oxi) ao menos uma vez na vida. $\mathrm{O}$ mesmo estudo informa que o Brasil representa $20 \%$ do consumo de cocaína/crack e o segundo maior mercado de crack do mundo.
Em números absolutos de usuários, a região sudeste abriga quase a metade dos usuários, com 1.4 milhões de indivíduos que usaram a droga no último ano.

O crack surgiu primeiramente nos Estados Unidos entre 1984 e 1985, fabricado por meio de processos caseiros para ser consumido em grupos. No Brasil, o primeiro relato de uso de crack data de 1989, na cidade de São Paulo (Nappo, Galduróz, \& Noto, 1996). 
O contexto de vulnerabilidade que permeia a vida dos usuários de crack pode ser exacerbado pelo uso compulsivo dessa substância, fazendo com que muitos se exponham a outras situações agravantes para a saúde. Nesse sentido, no Brasil e no exterior, alguns estudos realizados com usuários de crack encontraram perfis bastante similares, caracterizados principalmente por maioria do sexo masculino, jovens e de baixo nível educacional (Falck, Wang, \& Carlson, 2007; Laranjeira \& Ribeiro, 2010; Pulcherio, Stolf, Pettenon, Fensterseifer, \& Kessler, 2010).

De acordo com os dados da United Nations Office on Drugs and Crime (UNODC, 2014), os países desenvolvidos têm diminuído o consumo de cocaína, porém nos países em desenvolvimento, como o Brasil, o consumo tem aumentado.

Atualmente, mesmo com o último estudo nacional sobre o consumo de crack no Brasil (Bastos \& Bertoni, 2014b), ainda não há consenso a respeito dos insumos necessários para uma eficaz ação preventiva, capaz de minimizar a propagação de diversas doenças infectocontagiosas associadas ao consumo dessa substância (Fischer \& Coghlan, 2007; Ribeiro, Dunn, Sesso, Dias, \& Laranjeira, 2006). Tampouco uma diretriz eficaz de saúde pública, que possa auxiliar na solução dos problemas decorrentes do consumo abusivo e dos agravos à saúde dos usuários.

Tal constatação parece apontar a necessidade de mais investigações, que possam contribuir para aprofundar o conhecimento acerca de quem são esses usuários e seus contextos de vida, dentre outros fatores. Neste contexto, este estudo descreve os usuários de crack do município de Santos, cidade litorânea do Estado de São Paulo. A cidade abriga o maior porto da América Latina, com navios de rotas nacionais e internacionais, além de ser um corredor de transporte terrestre, por meio dos caminhões que circulam em seu território, vindos de diversos locais do Brasil e rotas do Mercosul. Essas características fazem com que a cidade seja uma das rotas internacionais de drogas e por esta razão merece especial atenção da saúde pública (Lacerda, Stall, Gravato, Tellini, \& Hudes, 1996).
Diversos estudos com populações de usuários de drogas injetáveis, caminhoneiros e trabalhadoras do sexo realizados em Santos revelaram a presença do consumo de crack em vários locais da cidade, apontando a necessidade de novas pesquisas nessa área (Mesquita et al., 2001; Silva, Pres, Morell, \& Areco, 2007; Villarinho et al., 2002). Além disto, estima-se que $19,8 \%$ dos residentes do município se encontram em situação de risco, distribuídos em favelas, cortiços e população de rua, acarretando maior vulnerabilidade nesta população (Morell, Brandão, \& Vázquez, 2012). Esses dados demonstram a necessidade de se estudar as características dos usuários de crack neste município. Este é um estudo inédito na região, com número relativamente elevado de participantes e que pode vir a contribuir para a elaboração e implementação de políticas públicas de saúde, voltadas para o melhor atendimento desses usuários.

\section{Método}

A pesquisa foi aprovada pelo Comitê de Ética em Pesquisa da Universidade Federal de São Paulo - UNIFESP (parecer CEP 1587/10).

Trata-se de estudo um transversal, descritivo e exploratório (Gil, 2008), de abordagem quantitativa.

\section{Amostra}

Participaram 292 sujeitos, usuários de crack ou ex-usuários, que estavam ou não em tratamento no momento da entrevista, de ambos os gêneros, com 18 anos ou mais. Foram considerados usuários os que estivessem consumindo a droga e, ex-usuários, os que estavam em abstinência por no mínimo seis meses. Todos deveriam ter consumido crack ao menos 25 vezes na vida. A eleição deste ponto de corte foi realizada com o intuito de se incluir apenas usuários que elegem a droga para o consumo e não aqueles que a usam por mera experimentação (Siegel, 1995). Foram excluídos usuários que estavam sob efeito da droga ou com sintomas de abstinência no momento da coleta. A amostragem foi intencional, definida pela 
técnica da bola de neve, utilizada para conseguir contato com sujeitos de difícil acessibilidade (Biernacki \& Waldorf, 1981).

\section{Procedimentos}

Foram localizados usuários, ex-usuários de crack e profissionais de saúde (informantes-chave), que serviram de contato, indicando outros usuários a serem entrevistados e assim sucessivamente (Oliveira \& Nappo, 2008a).

\section{Locais de Coleta de Dados.}

1. Centro de Atenção Psicossocial Álcool e Drogas do município de Santos (CAPSad) para a realização de entrevistas com os usuários em tratamento naquele equipamento.

2. Duas Organizações não Governamentais (ONGs) que prestam assistência e cuidados a moradores de rua e pessoas em situação de vulnerabilidade, propiciando atividades que incentivam o lazer e a reinserção social por meio de oficinas. Uma delas pertence a uma igreja evangélica e se propõe a tratar os usuários de substâncias psicoativas a partir do enfoque religioso. Nessas instituições foram entrevistados usuários que participavam das atividades ali oferecidas.

3. Nas ruas, em locais de concentração de usuários de crack.

\section{Instrumento}

O Instrumento utilizado foi uma versão adaptada do ASI - Addiction Severity Index, que foi traduzido para o português e publicado por Formigoni e Castel (1999). A adaptação do instrumento foi realizada em função da necessidade de mais questões específicas sobre o consumo de crack, assim como para se ter acesso a usuários que não se encontravam em situação de tratamento. Para essa adaptação foi realizado um estudo piloto com 20 usuários, que demonstraram bom entendimento das questões acrescentadas ao instrumento original.

O ASI é uma entrevista semiestruturada, com bons índices de validade e confiabilidade (Kosten, Rounsaville, \& Kleber, 1983). Fornece, além de dados socioeconômicos e demográficos, nível de problemas e necessidade de tratamento associados ao uso de substâncias psicoativas, avaliando sete áreas: condição médica, situação ocupacional, uso de álcool e outras drogas, situação legal, problemas psiquiátricos, familiares e sociais.

Os dados foram analisados por meio de análises descritivas (média, desvio padrão e frequência).

\section{Resultados}

Os dados econômicos e sociodemográficos estão descritos na Tabela 1.

$\mathrm{O}$ perfil dos investigados indica maior prevalência de sujeitos do sexo masculino, solteiros, pardos, moradores de rua, com idade média de 35 anos e escolaridade média de 8 anos. A maior parte da fonte de renda desses usuários era proveniente de atividades informais e a média de tempo de vínculo formal de trabalho foi de quatro anos, sendo que nos últimos 30 dias, o número de dias trabalhados foram nove em média

A Tabela 2 mostra o histórico de tratamento e sintomas psicológicos e psiquiátricos dos usuários de crack na vida e nos últimos 30 dias, bem como a permanência em ambiente controlado por igual período. Os sintomas psicológicos e psiquiátricos foram frequentemente mencionados pelos usuários entrevistados. No entanto, a maioria dos participantes não recebeu qualquer intervenção ou tratamento psicológico/psiquiátrico $(65,8 \%)$ e apenas 3,8\% recebiam pensão por incapacidade psiquiátrica. 
Tabela 1

Características Sócio-Demográficas e Econômicas dos Usuários de Crack no Município de Santos

\begin{tabular}{|c|c|c|}
\hline \multirow{2}{*}{ Variáveis } & \multicolumn{2}{|c|}{ Frequência } \\
\hline & $N$ & $(\%)$ \\
\hline \multicolumn{3}{|l|}{ Gênero } \\
\hline Masculino & 247 & 84,6 \\
\hline Feminino & 45 & 15,4 \\
\hline \multicolumn{3}{|l|}{ Raça } \\
\hline Parda & 123 & 42,1 \\
\hline Branca & 120 & 41,1 \\
\hline Negra & 48 & 16,4 \\
\hline Sem informação & 1 & 0,4 \\
\hline \multicolumn{3}{|l|}{ Estado civil } \\
\hline Solteiro & 144 & 49,3 \\
\hline Separado & 81 & 27,7 \\
\hline Casado & 57 & 19,5 \\
\hline Sem informação & 10 & 3,5 \\
\hline \multicolumn{3}{|l|}{ Situação de moradia habitual (últimos 3 anos) } \\
\hline Rua & 139 & 47,7 \\
\hline Com família ou companheiro & 82 & 28,1 \\
\hline Sozinho & 49 & 16,7 \\
\hline Com amigos & 14 & 4,8 \\
\hline Sem informação & 8 & 2,7 \\
\hline \multicolumn{3}{|l|}{ Padrão de trabalho habitual (últimos 3 anos) } \\
\hline Desemprego & 125 & 42,8 \\
\hline Trabalho Formal & 113 & 38,7 \\
\hline Meio período (irregulares) & 33 & 11,3 \\
\hline Aposentado/incapacitado & 15 & 5,1 \\
\hline Sem informação & 6 & 2,1 \\
\hline \multicolumn{3}{|l|}{ Fonte de renda no último mês } \\
\hline Informal & 95 & 32,5 \\
\hline Ilegal & 60 & 20,5 \\
\hline Emprego & 49 & 16,8 \\
\hline Não respondeu a fonte & 47 & 16,1 \\
\hline Pensões e benefícios & 22 & 7,5 \\
\hline Companheiro/família & 14 & 4,8 \\
\hline Outros & 4 & 1,4 \\
\hline Sem informação & 1 & 0,4 \\
\hline $\begin{array}{l}\text { Idade } \\
\text { Média (desvio-padrão) }\end{array}$ & \multicolumn{2}{|c|}{$34,8(9,3)$} \\
\hline $\begin{array}{l}\text { Anos completos de estudo } \\
\text { Média (desvio-padrão) }\end{array}$ & \multicolumn{2}{|c|}{$7,84(3,56)$} \\
\hline $\begin{array}{l}\text { Quanto tempo está vivendo nessa condição de moradia (anos) } \\
\text { Média (desvio-padrão) }\end{array}$ & \multicolumn{2}{|c|}{$5,32(7,7)$} \\
\hline $\begin{array}{l}\text { Quanto tempo durou o trabalho no qual você ficou mais tempo (anos) } \\
\text { Média (desvio-padrão) }\end{array}$ & \multicolumn{2}{|c|}{$4,44(4,71)$} \\
\hline $\begin{array}{l}\text { Quantos dias foi pago para trabalhar nos últimos } 30 \text { dias } \\
\text { Média(desvio-padrão) }\end{array}$ & \multicolumn{2}{|c|}{$9,84(11,86)$} \\
\hline
\end{tabular}


Tabela 2

Histórico de Tratamento e Sintomas Psicológicos e Psiquiátricos dos Usuários de Crack do Município de Santos na Vida e nos Últimos 30 Dias

Variáveis

Frequência

$\begin{array}{ll}N & (\%)\end{array}$

Você esteve em ambiente controlado (internado) nos últimos 30 dias

Não

244

83,6

Cadeia/Prisão

7

2,4

Tratamento Médico, droga/álcool ou psiquiátrico

Sem informação

Você está tomando alguma medicação prescrita de forma regular por problema de saúde

Sim

66

Sem informação

$1 \quad 0,3$

Recebeu tratamento psicológico

Sim

Recebeu prescrição psicofarmacológica - 30 dias

Sim

Sem informação

Recebeu prescrição psicofarmacológica - vida

Sim

Sem informação

Sintomas (Sim)

Depressão grave - 30 dias

Depressão grave - vida

Ansiedade ou tensão grave - 30 dias

Ansiedade ou tensão grave - vida

$198 \quad 68$

Alucinações - 30dias

Alucinações - vida

Problemas de compreensão, concentração e memória - 30 dias

$135 \quad 46,3$

Problemas de compreensão, concentração e memória - vida

$150 \quad 51,5$

Comportamento violento - 30 dias

$65 \quad 22,3$

Comportamento violento - vida

Pensamento sério sobre suicídio - 30 dias

$69 \quad 23,7$

Pensamento sério sobre suicídio - vida

$161 \quad 55,3$

Tentativa de suicídio - 30 dias

Tentativa de suicídio - vida 
Tabela 3

História de Uso do Crack Relatada pelos Usuários de Crack do Município de Santos

Variáveis

Crack (início de uso por faixa etária)

$\begin{array}{lcc}\text { Até } 12 \text { anos } & 17 & 5,8 \\ 13 \text { a } 18 \text { anos } & 91 & 31,2 \\ 19 \text { a } 29 \text { anos } & 121 & 41,4 \\ 30 \text { a } 39 \text { anos } & 43 & 14,7 \\ 40 \text { anos ou mais } & 16 & 5,5 \\ \text { Sem informação } & 4 & 1,4\end{array}$

Forma de uso do crack

Pipado

Fumado

Sem informação

Drogas associadas ao crack

Nenhuma droga

Álcool

Maconha

Cocaína

Sedativos

Álcool + Maconha

Álcool + Cocaína 7,9

Álcool + Cocaína + Maconha 16,1

Cocaína + Maconha

Outras combinações diversas de drogas

Sem informação

O crack é sua principal substância

Sim

Em relação à forma de obtenção da primeira droga consumida na vida, a maioria informou tê-la ganho, sendo a curiosidade o maior propulsor para o uso.

Na Tabela 3 é apresentada a história de uso do crack relatada pelos usuários.

A menor idade para início de consumo de crack foi de 8 e a máxima de 59 anos.
Apenas 15,8\% consumiam somente o crack, os demais consumiam o crack associado a outras drogas.

A maioria fazia uso desta substância na forma "pipada". A quantidade de pedras consumidas variou de 2 a 150 pedras/dia e o valor da pedra entre $\mathrm{R} \$ 5,00$ e $\mathrm{R} \$ 10,00$.

A Tabela 4 apresenta características psicossociais e familiares dos usuários. 
Tabela 4

Características Psicossociais e Familiares dos Usuários de Crack do Município de Santos

Variáveis

Frequência

\begin{tabular}{lll}
\cline { 2 - 2 } & $N$ & $\%$ \\
\hline
\end{tabular}

Com quem passa a maior parte do tempo livre

$\begin{array}{lcr}\text { Família } & 50 & 17,1 \\ \text { Amigos } & 43 & 14,7 \\ \text { Sozinho } & 155 & 53,1 \\ \text { Outros usuários de crack } & 40 & 13,7 \\ \text { Sem informação } & 4 & 1,4\end{array}$

Quantos amigos íntimos possui

$\begin{array}{ll}\text { Zero } & 159\end{array}$

Um a dois

Três ou mais

Sem informação

$11 \quad 3,7$

Cometeu algum crime

Sim

Ficou preso na vida

$\operatorname{Sim}$

Sem informação

Em relação ao tempo livre, a maioria ficava sozinha e referia não ter vínculo de amizade.

Em relação à situação legal, a maioria já cometeu algum crime e mais da metade dos entrevistados já esteve preso. Os dados mostraram que $95,4 \%$ não mencionaram detenção nos últimos 30 dias, mas $19,7 \%$ estiveram envolvidos em atividades ilegais visando lucro, e, 25\% estiveram envolvidos em atividades ilegais visando à compra da droga.

\section{Discussão}

A problemática atual vivenciada no Brasil, referente ao consumo abusivo e compulsivo de crack, tem gerado vários questionamentos acerca do perfil desses usuários, assim como da melhor maneira de atender às suas necessidades. Alguns estudos nacionais investigaram o perfil de usuários de crack (Bastos \& Bertoni, 2014a, 2014b; Dualibi, Ribeiro, \& Laranjeira, 2008). No entanto, ainda existem poucos dados a respeito deste perfil, havendo ainda necessidade de estudos que favoreçam uma maior compreensão das características socioeconômica, demográfica, necessidades de saúde e padrão de consumo de crack, para uma melhor abordagem ao usuário. O diferencial deste estudo, além do acesso a um elevado número de sujeitos, é o fato de incluir usuários de distintos locais e em momentos diferentes no que se refere ao consumo de crack. Dessa forma, foi possível apreender a diversidade desse grupo em seus múltiplos cenários, ampliando a compreensão das características e necessidades dessa população, justamente pelo fato de não estar focado em um grupo ou local específico, como a maioria dos estudos nacionais (Horta, Horta, Rosset, \& Horta, 2011; Ribeiro et al., 2006; Vargens, Cruz, \& Santos, 2011). 
É importante ressaltar que a cidade de Santos possui características únicas no que se refere aos usuários de crack. Estes são facilmente identificados em vários bairros, utilizando a droga de forma explícita em locais de acesso e circulação de pessoas, evidenciando de certa forma uma grande quantidade de usuários nos espaços públicos, como também identificado no recente estudo nacional realizado pela Fundação Oswaldo Cruz (FIOCRUZ), que revelou esse cenário em outros locais do país (Bastos \& Bertoni, 2014b).

A maior frequência de homens do que de mulheres já foi relatada em outros estudos dessa natureza nos Estados Unidos (Lacerda et al., 1996) e no Brasil (Bastos \& Bertoni, 2014b). No último estudo da FIOCRUZ realizado no Brasil, com amostragem de 7.381 usuários de crack, $78,7 \%$ eram homens (Bastos \& Bertoni, 2014b). No estudo anterior realizado pelo Centro Brasileiro de Informações sobre Drogas - CEBRID (Carlini et al., 2007), em uma amostra de 430 usuários de crack, 60,7\% eram homens. Dados da literatura e do presente estudo sugerem haver questões de gênero envolvidas no consumo e acesso à droga, em que as mulheres usuárias conseguem mais frequentemente o crack a partir do companheiro ou de outros homens, não se expondo a maiores riscos, tornando o acesso a elas mais difícil (Nappo et al., 2004).

Em relação à idade média encontrada nesta pesquisa, nota-se que está mais alta do que as relatadas nos estudos nacionais da década de 1990, quando o crack foi apreendido pela primeira vez no Brasil (Nappo et al., 1996). Alguns estudos mais recentes indicam que a maioria dos usuários de crack é constituída por jovens adultos, com idade média entre 25 e 30 anos (Bastos \& Beroni, 2014b; Freire, Santos, Bortolini, Moraes, \& Oliveira, 2012; Horta et al., 2011). No entanto, também já se constatou média de idade mais próxima à encontrada no presente estudo (Dias, Araújo, \& Laranjeira, 2011; Vargens et al., 2011). Esses dados podem revelar que muitos dos atuais usuários de crack já o eram quando a droga foi detectada no Brasil. Além disto, o perfil desses usuários parece estar se modificando no decorrer do tempo, visto que no presente estudo a idade média de início de consumo dessa substância foi de 23,1 anos, ou seja, já na vida adulta.

Apesar da idade média de início de consumo de crack ter ocorrido na fase adulta, chama a atenção a variação encontrada, sendo a mais baixa 8 anos, e a mais alta, 59 anos. Somando-se a esse dado, a curiosidade foi o motivo mais citado para se iniciar esse consumo, mesmo dado encontrado no estudo nacional realizado pela FIOCRUZ (Bastos \& Bertoni, 2014b). A curiosidade como fator que propulsiona à experimentação do uso de crack evidencia a importância de se reforçar as intervenções de prevenção ao risco associado ao uso desta droga, mesmo em situações experimentais (Bastos \& Bertoni, 2014b). Medidas preventivas devem ocorrer focadas, principalmente, na maior disponibilidade de informações sobre a droga e os malefícios decorrentes de seu uso compulsivo, o qual, mesmo em curto prazo, pode comprometer a vida do usuário. Sugere-se que tais intervenções sejam de cunho educativo, valorizando as experiências de vida dos sujeitos e fundamentadas na ética e nos direitos humanos. As intervenções educativas precisam considerar também que muitos iniciaram o uso de substâncias psicoativas por outras drogas, por isso ingressam no uso de crack já na fase adulta. O início do uso de drogas se deu principalmente com álcool e maconha e os usuários foram ou ainda são consumidores dessas e de outras substâncias. No presente estudo, apenas $15,8 \%$ consumiam exclusivamente crack, os demais eram poliusuários, o que torna o tratamento e a adesão mais difíceis e complexos, além de poder retardar a recuperação e a reinserção social do usuário (Oliveira \& Nappo, 2008b).

Em relação à escolaridade, os usuários de crack pesquisados variaram desde aqueles sem nenhuma escolaridade (analfabetos) até os que possuíam formação universitária. No entanto, a média de tempo estudado foi relativamente baixa, um problema comum entre esta população, considerando sua dificuldade em permanecer na escola (Bastos \& Bertoni, 2014b; Dualibi et al., 2008; Pechansky, Szobot, \& Schivoletto, 2004; Pulcherio et al., 2010). Deve-se considerar ainda que muitos usuários se desenvolveram em ambientes familiares caracterizados por ausência 
de figura paterna e famílias caracterizadas por uma estruturação frágil, permeadas por violência doméstica e vitimização infantil (Narvaez, et al., 2015). Tais fatores são prevalentes nesta população e podem ser considerados fatores de risco para o uso de drogas (Huang et al., 2011; Shin, Miller. \& Teicher, 2013; Tucci, Kerr-Corrêa, \& Souza-Formigoni, 2010). Além disto, as situações de violência intrafamiliar, pobreza e desigualdade social, presentes entre os usuários de drogas, especialmente entre aqueles que fazem uso de crack, podem também favorecer o trabalho infantil, conduzindo muitos deles à evasão escolar em idade precoce (Marchi, 2013). Assim, a desestrutura familiar, por diferentes razões, é um dos fatores que afeta diretamente tanto a condição de aprendizagem quanto a permanência escolar.

A maior prevalência neste estudo foi de pessoas solteiras e que moravam na rua. A situação conjugal foi similar à encontrada no estudo nacional da FIOCRUZ (Bastos \& Bertoni, 2014b), no qual os solteiros constituíam $60,6 \%$ da população estudada. Estudos realizados em outros países e o recente estudo nacional também encontraram uma alta prevalência de moradores de rua entre os usuários de crack (Bastos \& Bertoni, 2014b; Fischer et al., 2006; Prinzleve et al., 2004). No presente estudo a frequência de pessoas que moravam na rua foi maior do que a encontrada no estudo nacional, respectivamente $47,7 \%$ e $40 \%$. O fato de Santos ser uma cidade turística, possuir o maior porto da América Latina, estar próxima à capital do estado de São Paulo e de Cubatão, importante polo industrial, pode favorecer a migração de pessoas originárias de outras localidades. Estas pessoas, muitas vezes, acabam por se fixar na cidade, nos locais de maior vulnerabilidade, onde os valores de locação de imóveis são mais baixos, ou podem se tornar moradores de/na rua por dificuldade de retorno ao seu local de origem, aumentando a vulnerabilidade para o consumo de drogas, entre essas, o crack.

Embora alguns usuários tenham informado passar seu tempo livre com a família ou amigos, chama a atenção o isolamento social referido pela maioria desses indivíduos. A relação com a droga muitas vezes acaba por estabelecer um padrão no qual apenas quem faz parte das cenas de uso passa a ter espaço na vida do usuário e a obtenção da droga passa a ser o maior sentido e incentivo para sua sobrevivência (Chaves, Sanchez, Ribeiro, \& Nappo, 2011). Esses dados podem sugerir que uma das consequências do uso crônico e compulsivo de crack é a manutenção de vínculos basicamente constituídos por pessoas que também são usuárias da substância, levando-os a maior isolamento social.

Nos últimos três anos, $39 \%$ dos entrevistados relataram ter trabalho formal; já no último mês a queda foi acentuada, apenas $16,8 \%$ ainda o mantinham. Além disto, outro dado que chama a atenção é que o número médio de dias de trabalho nos últimos trinta dias foi de 9 dias, revelando a dificuldade do usuário em permanecer no ambiente de trabalho, provavelmente devido à dependência ou uso compulsivo da substância. Como consequência da dificuldade de se manterem com vínculo de trabalho, os dados revelaram que nos últimos 30 dias, 19,7\% estiveram envolvidos em atividades ilegais visando lucro e $25 \%$ visando a compra da droga.

A grande proporção de usuários que está ou já esteve envolvido em situações ilegais, dentre essas o tráfico, que em geral figura como principal meio de obtenção do dinheiro ou da droga e acaba por aprisionar o usuário; aumenta a dificuldade de se romper o ciclo de consumo, mesmo quando o usuário tem desejo de controlar seu uso ou abster-se da substância (Oliveira \& Nappo, 2008a)

A quantidade e frequência de sintomas psicológicos/psiquiátricos referidos pelos usuários revelaram que o uso abusivo de crack gera intenso sofrimento emocional nesses indivíduos. Os usuários referiram alta frequência de sintomas de ansiedade, depressão, pensamento suicida, problemas de concentração e memória, quadro preocupante no universo desses sujeitos. Em contrapartida, chama a atenção os dados relativos à ausência de tratamento clínico, psiquiátrico e psicológico recebido; mostrando que a grande maioria não acessou e também não recebeu assistência à saúde, além de revelar a provável existência de dificuldade em acessar os ser- 
viços disponíveis e aderirem quando conseguem chegar até estes.

Diante da baixa adesão ao tratamento e do pouco acesso aos serviços da rede de atendimento aos usuários de álcool ou outras drogas, constatados entre os usuários do presente estudo e entre os usuários de crack em geral (Bastos \& Bertoni, 2014b), as intervenções baseadas nas Estratégias de Redução de Danos, feitas in loco com objetivo mais educativo, associado ao desenvolvimento de práticas mais seguras de sexo e uso de drogas, podem ser formas viáveis de se conseguir a inserção junto a essa população e se reduzir os danos (Moraes, 2010). Proposta de intervenção também interessante, como o consultório na rua, que leva o atendimento psicossocial e a testagem rápida para DST/AIDS aos locais de concentração de usuários, por meio de uma equipe especializada, pode ser indicada para essa população, pois favorece o acesso a informações e a insumos, como preservativos, atraindo e favorecendo práticas de autocuidado (Moraes, 2010). A atuação do consultório na rua pode ainda fortalecer a autoestima dos usuários, estabelecer vínculos e servir de ponte entre os mesmos, os serviços de saúde e outras redes de cuidados, por estabelecer uma dinâmica em que o atendimento in loco é adequado à necessidade e perfil desse usuário (Jorge \& Corradi-Webster, 2012). No entanto, no Brasil, o consultório na rua ainda se encontra em fase de implantação em muitos municípios e são necessários ainda estudos que avaliem os resultados das intervenções propostas para este equipamento junto a esta população.

O consumo de álcool associado ao uso de crack tem sido relatado em vários estudos nacionais e internacionais. No presente estudo, tal associação foi a mais referida pelos usuários, dado também encontrado no recente estudo nacional da FIOCRUZ (Bastos \& Bertoni, 2014b). O uso de álcool entre usuários de crack ocorre principalmente no final de um padrão de consumo compulsivo da droga, quando os mesmos se encontram exaustos pela falta de ingestão de alimentos, privação de sono e falta de cuidados pessoais (Chaves et al., 2011; Gossop, Manning, \& Ridge, 2006; Ribeiro, Sanchez, \& Nappo, 2010).
O uso concomitante de álcool e crack pode reduzir os prejuízos imediatos pela interrupção do consumo de crack após um consumo compulsivo dessa substância. No entanto, o efeito combinado dessas duas substâncias em longo prazo pode ser mais prejudicial e conduzir à dependência de álcool e outros problemas relacionados a esse uso concomitante (UNODC, 2010).

A maconha aparece como a segunda droga mais associada ao consumo de crack. A maconha, além de diminuir a compulsão e fissura, traz outros benefícios, como a "larica", vontade de comer muitas vezes compulsivamente após seu consumo, possibilitando também que o usuário consiga relaxar, dormir e manter os cuidados básicos de higiene (Chaves et al., 2011). O consumo de maconha por usuários de crack pode ser entendido como uma estratégia de redução de danos desenvolvida pelos próprios usuários. No entanto, o presente estudo não avaliou os efeitos do uso concomitante dessas substâncias e aponta a necessidade de estudos que verifiquem se tais efeitos conduziriam ao uso mais controlado de crack, contribuindo para a minimização dos danos e riscos causados pelo consumo compulsivo dessa droga.

Outro dado preocupante e que merece maior atenção nas ações de saúde voltadas para essa população é que a forma de consumo de crack mais utilizada e difundida entre os usuários foi a "pipada" (73,2\%), dado similar ao encontrado no estudo nacional da FIOCRUZ (Bastos \& Bertoni, 2014b). Nessa forma, o crack é consumido em diversos tipos de cachimbos confeccionados pelos próprios usuários, os quais na grande maioria das vezes são compartilhados, aumentando o risco de infecções por Doenças Sexualmente Transmissíveis e HIV (Fischer \& Coghlan, 2007; Oliveira \& Nappo, 2008a). Esse seria um outro aspecto a ser discutido com os usuários nos locais de consumo da droga, preferencialmente por equipes que constituem o consultório na rua.

Os dados desse estudo apontam grande variação na quantidade de pedras de crack consumidas e frequência de uso, as quais muitas vezes estão vinculadas à facilidade de acesso, quantidade de dinheiro disponível para compra, envolvimento com o tráfico, troca de sexo pela droga e 
a própria resistência física e psicológica de seus consumidores. Alguns usuários conseguem estabelecer o uso de crack em alguns dias da semana e em quantidade pré-definida por eles, sugerindo que existe possibilidade de consumo dessa substância com determinado controle. Porém, o padrão compulsivo de consumo de crack tem sido o mais relatado na literatura (Dias et al., 2011).

Contrariando a imagem social do usuário de crack, neste estudo, eles foram de grande auxílio na coleta dos dados, uma vez que acompanharam e protegeram as entrevistadoras no campo, apresentando ainda novos usuários. Portanto, a visão do senso comum, difundida pela mídia em relação aos usuários como pessoas nocivas e agressivas (Romanini \& Rosso, 2012), não se confirmou durante as idas ao campo, ao contrário, trouxeram para a equipe a reflexão sobre o pânico moral instalado em nossa sociedade em relação a essa população. Segundo Romanini e Roso (2012), a mídia tem descrito o fenômeno do crack por meio de diversas estratégias ideológicas que obscurecem a compreensão do fenômeno em questão, colaborando com a manutenção de situações de exclusão, responsabilizando o usuário pelo aparecimento e pela solução do seu problema e prejudicando o seu acesso aos serviços de saúde. A atuação da mídia também tem favorecido o estigma e o preconceito entre os profissionais de saúde que deveriam acolher e cuidar desta população, o que aumenta ainda mais as dificuldades de acesso aos serviços da rede (Ronzani, Noto, \& Silveira, 2014).

A escuta e o reconhecimento desses usuários como cidadãos com direitos e deveres devem ser vistos a partir de um devido acolhimento, no qual haja corresponsabilidade dos atos e das escolhas, tanto para a busca quanto para a adesão ao tratamento. O tratamento desses usuários deve compreender esse acolhimento inicial, sendo necessário respeitar as diferenças e se construir um plano terapêutico individualizado, por meio do qual se priorize as necessidades subjetivas do usuário. Nesse sentido, os dados do presente estudo reforçam a necessidade de implantação em maior escala das intervenções propostas para o consultório na rua (Moraes, 2010). Esta intervenção vem ao encontro das necessi- dades dos usuários que não conseguem ou não querem chegar aos serviços de saúde, mas que apresentam, muitas vezes, necessidades básicas de saúde.

A técnica bola de neve, apesar de favorecer o acesso a populações que seriam de difícil acesso, trouxe como limitação a este estudo a presença de muitos usuários que se encontravam em situação de rua. Nesse sentido, essa característica suscitou questões mais complexas que não puderam ser descritas neste estudo. Por exemplo, a qualidade e grau de vínculo destes usuários com as diferentes instituições podem ter influenciado no perfil detectado no presente estudo, assim como nas formas mais viáveis de atendimento a ser prestado a essa população. Desta forma, este estudo revelou um cotidiano destes usuários bastante precarizado, marcado por alta vulnerabilidade social, sugerindo outros estudos que, associados à metodologia de acesso a esses usuários em seus locais de consumo, possam aprofundar aspectos da dinâmica psicossocial daqueles que se encontram em situação de rua. Inclusive, cabe destacar que o presente estudo não teve acesso à população de usuários em tratamento em clínicas privadas, assim como às classes sociais mais elevadas, bem como limitado acesso às mulheres que não foram suficientemente representadas na população estudada. Portanto, outros estudos precisam investigar melhor o envolvimento do crack na vida desses usuários.

Apesar das limitações da técnica da bola de neve, o perfil de usuários de crack encontrado no presente estudo foi similar ao recente estudo da FIOCRUZ (Bastos \& Bertoni, 2014b) que se utilizou de metodologia distinta da realizada nesse estudo, sugerindo que o método aqui utilizado também foi adequado.

Pode-se concluir a partir dos achados deste estudo que o perfil dos usuários de crack investigados é constituído por uma maioria de homens; com baixa escolaridade; praticantes de atividades ilegais e fonte de renda proveniente de atividades informais; moradores de rua; solteiros; pardos; com frágil vínculo familiar e social; significativa presença de sintomas psicológicos e psiquiátricos; sem histórico de tratamento psicológico/psiquiátrico ao longo da vida; poli- 
-usuários de substâncias, sendo o crack a droga de preferência. A constatação de tal perfil pode indicar que as intervenções fundamentadas nas Estratégias de Redução de Danos feitas in loco, com objetivo educativo e preventivo, podem ser as formas mais adequadas de se conseguir inserção e reduzir os danos nesta população, principalmente aqueles que se encontram em situação de rua. Assim, tais achados podem auxiliar e fortalecer políticas públicas como o consultório na rua, especialmente na área de álcool ou outras drogas.

\section{Referências}

Bastos, F. I., \& Bertoni, N. (2014a). Pesquisa Nacional sobre o uso de crack: Quem são os usuários de crack/ou similares do Brasil? Quantos são nas capitais brasileiras? Rio de Janeiro, RJ: Editora da Fundação Oswaldo Cruz. Recuperado em http://www.obid.senad.gov.br/ portais/OBID/biblioteca/documentos/Relatorios/329786.pdf

Bastos, F. I., \& Bertoni, N. (2014b). Quem são os usuários de crack e/ou similares do Brasil? Perfil sociodemográfico e comportamental destes usuários: Resultados de uma pesquisa de abrangência nacional. In F. I. Bastos \& N. Bertoni, Pesquisa Nacional sobre o uso de crack: Quem são os usuários de crack/ou similares do Brasil? Quantos são nas capitais brasileiras? Rio de Janeiro, RJ: Editora da Fundação Oswaldo Cruz. Recuperado em http://www.obid.senad.gov.br/ portais/OBID/biblioteca/documentos/Relatorios/329786.pdf

Biernacki, P., \& Waldorf, D. (1981). Snowball sampling. Sociological Methods and Research, 5(2), 141-163.

Carlini, E. A., Galduróz, J. C., Noto, A. R., Carlini, C. M., Oliveira, L. G., Nappo, A. S., ...Sanchez, Z. V. D. M. (2007). II Levantamento domiciliar sobre o uso de drogas psicotrópicas no Brasil: Estudo envolvendo as 108 maiores cidades do país - 2005. São Paulo, SP: Páginas \& Letras.

Chaves, T. V., Sanchez, Z. M., Ribeiro, L. A., \& Nappo, A. S. (2011). Fissura por crack: Comportamentos e estratégias de controle de usuários e ex-usuários. Revista de Saúde Pública, 45(6), 1168-1175. doi:10.1590/S003489102011005000066
Dias, A. C., Araújo, M. R., \& Laranjeira, R. (2011). Evolução do consumo de crack em coorte com histórico de tratamento. Revista de Saúde Pública, 45(5), 938-948. doi:10.1590/S003489102011005000049

Dualibi, L. B., Ribeiro, M., \& Laranjeira, R. (2008). Profile of cocaine and crack users in Brazil. $\mathrm{Ca}$ dernos de Saúde Pública, 4(Supl. 24), 545-557. doi:10.1590/S0102-311X2008001600007

Falck, R. S., Wang, J., \& Carlson, R. G. (2007). Crack cocaine trajectories among users in a Midwestern American city. Addiction, 102(9), 14211431. doi:10.1111/j.1360-0443.2007.01915.x

Fischer, B., \& Coghlan, M. (2007). Crack use in North American cities: The neglected 'epidemic'. Addiction, 102, 1340-1341. doi:10.1111/ j.1360-0443.2007.01963.x

Fischer, B., Rehm, J., Patra, J., Kalousek, K., Haydon, E., Tyndall, M., \& El-Guebaly, N. (2006). Crack across Canada: Comparing crack users and crack non-users in a Canadian multi-city cohort of illicit opioid users. Addiction, 101, 1760-1770. doi:10.1111/j.1360-0443.2006.01614.x

Formigoni, M. L. O. S., \& Castel, S. (1999). Escalas de avaliação de dependência de drogas: Aspectos gerais. Revista de Psiquiatria Clínica, 26(1), 5-31.

Freire, S. D., Santos, P. L., Bortolini, M., Moraes, J. F. D., \& Oliveira, M. S. (2012). Intensidade de uso de crack de acordo com a classe econômica de usuários internados na cidade de Porto Alegre/Brasil. Jornal Brasileiro de Psiquiatria, 61(4), 221-226. doi:10.1590/S004720852012000400005

Gil, A. C. (2008). Métodos e técnicas de Pesquisa Social (6. ed.). São Paulo, SP: Atlas.

Gossop, M., Manning, V., \& Ridge, G. (2006). Concurrent use of alcohol and cocaine: Differences in patterns of use and problems among users of crack cocaine and cocaine powder. Alcohol Alcohol, 41(2), 121-125. doi:10.1111/j.13600443.2006.01497.x

Horta, R. L., Horta, B. L., Rosset, A. P., \& Horta, C. L. (2011). Perfil dos usuários de crack que buscam atendimento em Centros de Atenção Psicossocial. Cadernos de Saúde Pública, 27(11), 22632270. doi:10.1590/S0102-311X2011001100019

Huang, S., Trapido, E., Fleming, L., Arheart, K., Crandall, L., French, M., \& Prado, G. (2011). The long-term effects of childhood maltreat- 
ment experiences on subsequent illicit drug use and drug-related problems in young adulthood. Addictive Behaviors, 36(1-2), 95-102. doi:10.1016/j.addbeh.2010.09.001

Jorge, J. S., \& Corradi-Webster, C. M. (2012). Consultório de Rua: Contribuições e desafios de uma prática em construção. Saúde e Transformação Social, 3(1), 39-48. Recuperado em http://www. redalyc.org/pdf/2653/265322710007.pdf

Kosten, T. R., Rounsaville, B. J., \& Kleber, H. D. (1983). Concurrent validity of the addiction severity index. Journal of Nervous \& Mental Disease, 171, 606-610.

Lacerda, R., Stall, R., Gravato, N., Tellini, R., \& Hudes, S. (1996). HIV infection and risk behavior among male port workers in Santos, Brazil. American Journal of Public Health, 86, 11581160 .

Laranjeira, R., \& Ribeiro, M. (Eds.). (2010). O tratamento do usuário de crack: Avaliação clínica, psicossocial, neuropsicológica e de risco. Terapias psicológicas, farmacoterapia $e$ reabilitação. Ambientes de tratamento. São Paulo, SP: Casa Leitura Médica. Recuperado em http://portal.cnm.org.br/sites/9700/9797/ docBibliotecaVirtual/O_Tratamento_do_Usuario_de_crack.pdf

Laranjeira, R., Madruga, C. S., Ribeiro, M., Pinsky, I., Caetano, R., \& Mitsuhiro, S. S. (2012). II LE$N A D$ - II Levantamento de álcool e drogas. $O$ uso de cocaína e crack no Brasil. Recuperado em http://inpad.org.br/lenad/resultados/cocaina-e-crack/resultados-preliminares/

Marchi, R. C. (2013). Trabalho infantil: Representações sociais de sua instituição em Blumenau/SC. Educar em Revista, 47, 249-265. Recuperado em http://www.scielo.br/pdf/er/n47/13.pdf

Mesquita, F., Kral, A., Reingold, A., Bueno, R., Trigueiros, D., Araujo, P. J., \& Santos Metropolitan Region Collaborative Study Group. (2001). Trends of HIV Infection among injection drug users in Brazil in the 1990s: The impact of changes in patterns of drug use. Journal of Acquired Immune Deficiency Syndrome, 28(3), 298-302. Recuperado em http://journals.lww. com/jaids/toc/2001/11010

Moraes, M. (2010) Algumas implicações da ética punitiva e da ética fragmentária para as lutas contra-hegemônicas no campo das políticas públicas sobre drogas: Na trilha das provocações atrevidas de Roberto Éfrem Filho. In B. Medra- do, J. Lyra, M. Azevedo, \& J. Brasilino (Eds.), Homens e masculinidades: Práticas de intimidade e políticas públicas (pp. 167-176). Recife, PE: Instituto PAPAI. Recuperado em http:// www.unfpa.org.br/Arquivos/homens_masculinidades.pdf

Morell, M. G. G., Brandão, M. V. M., \& Vázquez, D. A. (2012). Os Estados e as Regiões Metropolitanas constitutivas do Observatório das Metrópoles no Censo 2010. Observatório das Metrópoles, Instituto Nacional de Ciências e Tecnologia. Recuperado em http://web.observatoriodasmetropoles.net/download/Os_Estados_e_as_RMs no_Censo_2010.pdf

Nappo, S. A., Galduróz, J. C., \& Noto, A. R. (1996). Crack use in São Paulo. Substance Use \& Misuse, 31(5), 565-579.

Nappo, A. S., Sanchez, Z. V. D. M., Oliveira, L. G., Santos, A. S., Coaradete, J., Jr., Pacca, J. C. B., \& Lacks, V. (2004). Comportamento de risco de mulheres usuárias de crack em relação às DST/ Aids. São Paulo, SP: Centro Brasileiro de Informações sobre Drogas Psicotrópicas.

Narvaez, J. C. M., Pechansky, F., Jansen, K., Pinheiro, R. T., Silva, R. A., Kapczinski, F., \& Magalhães, P. V. (2015). Quality of life, social functioning, family structure, and treatment history associated with crack cocaine use in youth from the general population. Revista Brasileira de Psiquiatria, 37, 211-218. doi:10.1590/15164446-2014-1494

Oliveira, L., \& Nappo, A. S. (2008a). Crack na cidade de São Paulo: Acessibilidade, estratégias de mercado e formas de uso. Revista de Psiquiatria Clínica, 35(6), 212-218. doi:10.1590/S010160832008000600002

Oliveira, L., \& Nappo, A. S. (2008b). Caracterização da cultura de crack na cidade de São Paulo: Padrão de uso controlado. Revista de Saúde Pública, 42(4), 664-671. doi:10.1590/S003489102008005000039

Pechansky, F., Szobot, C. M., \& Schivoletto. S. (2004). Uso de álcool em adolescentes: Conceitos, características epidemiológicas e fatores etiopatogênicos. Revista Brasileira de Psiquiatria, 26, 14-17. doi:10.1590/S151644462004000500005

Prinzleve, M., Haasen, C., Zurhold, H., Matali, J. L., Bruguera, E., Gerevich, J., ...Krausz, M. (2004). Cocaine use in Europe - A multi-centre study: Patterns of use in different groups. Eu- 
ropean Addiction Research, 10(4), 147-155. doi:10.1159/000079835

Pulcherio, G., Stolf, A. R., Pettenon, M., Fensterseifer, D. P., \& Kessler, F. (2010). Crack - Da pedra ao tratamento. Revista da AMRIGS, 54(3), 337-343. Recuperado em http://amrigs.org.br/ revista/54-03/018-610 crack NOVO.pdf

Ribeiro, L. A., Sanchez, Z. M., \& Nappo, S. A. (2010). Surviving crack: A qualitative study of the strategies and tactics developed by Brazilian users to deal with the risks associated with the drug. BMC Public Health, 10, 671. doi:10.1186/14712458-10-671

Ribeiro, M., Dunn, J., Sesso, R., Dias, C. A., \& Laranjeira, R. (2006). Causes of death among crack cocaine users. Revista Brasileira de Psiquiatria, 28(3), 196-202. doi:10.1590/S151644462006000300010

Romanini, M., \& Rosso, A. (2012). Mídia e Crack: Promovendo saúde ou reforçando relações de dominação? Psicologia Ciência e Profissão, 32(1), 82-97. doi:10.1590/S1414-98932012000100007

Ronzani, T. M., Noto, A. R., \& Silveira, P. S. (2014). Reduzindo o estigma entre usuários de drogas: Guia para profissionais e gestores. Juiz de Fora, MG: Editora da Universidade Federal de Juiz de Fora.

Shin, A. H., Miller, D. P., \& Teicher, M. H. (2013). Exposure to childhood neglect and physical abuse and developmental trajectories of heavy episodic drinking from early adolescence into young adulthood. Drug Alcohol and Dependence, 127, 31-38. doi:10.1016/j.drugalcdep.2012.06.005

Siegel, R. K. (1995). New patterns of cocaine use: Changing doses and routes. In N. Kozel \& E. H. Adams (Eds.), Cocaine use in America: Epidemiologic and clinical perspective. Rockville, MD: National Institute on Drug Abuse. Recuperado em https://archives.drugabuse.gov/pdf/ monographs/61.pdf

Silva, N. G., Pres, C. A., Morell, M. G. G. P., \& Areco, K. N. (2007). Fatores associados à infecção pelo HIV em trabalhadoras do sexo (TS) em Santos - SP. Saúde Coletiva, 4(18), 168172. Recuperado em http://www.redalyc.org/ pdf/842/84218403.pdf
Tucci, A. M., Kerr-Correa, F., \& Souza-Formigoni, M. L. (2010). Childhood trauma in substance use disorder and depression: An analysis by gender among a Brazilian clinical sample. Child Abuse and Neglect, 34(2), 95-104. doi:10.1016/j.chiabu.2009.07.001

United Nations Office on Drugs and Crime. (2010). World Drug Report (United Nations Publication, Sales No. E.10.XI.13). New York. Recuperado em http://www.unodoc.org/documents/ wdr/WDR_2010/World_Drug_Report_2010_ lo-res.pdf

United Nations Office on Drugs and Crime. (2014). World Drug Report (United Nations Publication, Sales No. E.14.XI.7). New York. Recuperado em https://www.unodc.org/documents/ wdr2014/World_Drug_Report_2014_web.pdf

Vargens, R. W., Cruz, M. S., \& Santos, M. A. (2011). Comparação entre usuários de crack e de outras drogas em serviço ambulatorial especializado de hospital universitário. Revista Latino-Americana de Enfermagem, 19, 804-812. doi:10.1590/ S0104-11692011000700019

Villarinho, L., Bezerra, I., Lacerda, R., Latorre, M. R. D., Paiva, V., Stall, R., \& Hearst, N. (2002). Caminhoneiros de rota curta e sua vulnerabilidade ao HIV, Santos, SP. Revista de Saúde Pública, 4(Supl. 36), 61-67. doi:10.1590/S003489102002000500009
Recebido: 26/10/2015

$1^{a}$ revisão: 12/04/2016 Aceite final: 14/04/2016 